\title{
Could sentinel lymph node biopsy be exempted for ductal carcinoma in situ after mastectomy?
}

\author{
Jiqiao Yang ${ }^{1} \cdot$ Qing Lv $^{1}$ (B)
}

Received: 19 June 2018 / Accepted: 7 July 2018 / Published online: 19 July 2018

(c) The Japanese Breast Cancer Society 2018

\section{To The Editor,}

I want to congratulate Dr. Watanabe et al. for their study on the upstaging to invasive ductal carcinoma (IDC) after mastectomy for ductal carcinoma in situ (DCIS) [1]. They reported that $22.6 \%$ of 226 DCIS lesions were upgraded to IDC after mastectomy, and the prevalence of macrometastasis of sentinel lymph nodes (SLNs) was $0.9 \%$. I would like to make two comments on this study. First, the authors believed SLN biopsy for patients undergoing mastectomy with a preoperative diagnosis of DCIS was overtreatment based on the fact that the prevalence of SLN metastasis was low and the long-term outcome was excellent. However, although the reported 5-year recurrence-free survival was as high as $99.5 \%$, I noticed the median follow-up period was only 31 months. The survival might be biased with a large ratio of censored data. Moreover, three out of five patients with SLN metastasis were post-operatively pathologically diagnosed as DCIS, indicating that the micro-invasive component were likely to be missed in the pathological test in this study, not only for original lesions, but also for SLNs. As a result, the rate of upstaging and SLN metastasis could both be underestimated. Second, since the surgical procedures were decided by patients themselves, I wonder how many of the included lesions could alternatively be treated by breast-conserving surgery and how many were lesions with a wide range of micro-calcifications or suspected intraductal spreadings. It would be essential to know if there were any differences in rates of upstaging and/or SLN metastasis between these two types of patients.

\section{Compliance with ethical standards}

Conflict of interest None of the authors has relevant conflicts of interest to declare.

\section{Reference}

1. Watanabe Y, Anan K, Saimura M, Koga K, Fujino M, Mine M, et al. Upstaging to invasive ductal carcinoma after mastectomy for ductal carcinoma in situ: predictive factors and role of sentinel lymph node biopsy. Breast Cancer. 2018. https://doi.org/10.1007/ s12282-018-0871-7.

This comment refers to the article available online at https://doi. org/10.1007/s12282-018-0871-7.

Qing Lv

lqlq1963@163.com

1 Department of Breast Surgery, West China Hospital, Sichuan University, Guoxuexiang 37, Chengdu 610041, People's Republic of China 